\title{
Significance of General Practitioners and Nurses Diagnostic Skills in Managing Delirium in Elderly Patients
}

\author{
Marzanna Derkacz-Jedynak, Andrzej Brodziak* and Alicja Różyk-Myrta \\ Institute of Medical Sciences, University of Applied Sciences in Nysa, Poland
}

*Corresponding author: Andrzej Brodziak, Institute of Medical Sciences, University of Applied Sciences in Nysa, Poland.

Received Date: December 03, 2018

Published Date: December 12, 2018

\begin{abstract}
Delirium consists of a specific impairment of consciousness which occurs in elderly patients due to the physiological deterioration of adaptive abilities and adverse effects of various stimuli. The incidence of delirium varies between reviewed populations and may be underestimated due to diagnostic difficulties, the specificity of geriatric conditions and insufficient knowledge among healthcare providers. Delirium is often confused with the behavioral and psychological symptoms of dementia (BPSD), depression and disorders secondary to "geriatric giants" or somatic illnesses. The diagnostic criteria of delirium are listed by the authors of ICD10 and DSM classifications. For general practitioners and nurses, proper diagnosis of delirium in elderly patients is a challenging process. Diagnosing delirium by non-qualified healthcare providers is facilitated by numerous psychometric and specialist tools and scales. The most common are: CAM - the Confusion Assessment Method, MDAS - the Memorial Delirium Assessment Scale; DOSS - the Delirium Observation Screening Scale and the recently verified tool 4AT-the rapid assessment test for delirium. Proper diagnosis of delirium warrants optimization of care and stress reduction by formal and informal caregivers.
\end{abstract}

Keywords: Delirium; Dementia; Management of delirium; Assessment tools for delirium

\section{Introduction}

Delirium consists of specific impairment of consciousness, which occurs in elderly patients due to the deterioration of adaptive abilities and adverse effects of various stimuli. Delirium worsens the outcome of care of patients, who are hospitalized, stay in different medical institutions or at home [1-6]. Healthcare providers should be familiar with the predisposing factors, diagnostic criteria and methods of managing delirium to be able to provide better prevention and treatment of the elderly. This will also lead to a reduction in burdens on health and social care providers $[7,8]$. Efficient and proper diagnosis of delirium in elderly patients is a challenge for general practitioners and nurses. The incidence of delirium may be underestimated due to diagnostic difficulties, the specificity of geriatric conditions and insufficient knowledge among healthcare providers [6].

\section{Discussion}

Attempts to estimate the incidence of delirium lead to varying results, depending on what population is being analyzed. The incidence of delirium vary also depends on the diagnostic criteria which have been used [5,6]. In the general geriatric population, the incidence of delirium does not exceed $2 \%$. According to the recent meta-analysis, the incidence of delirium in patients on geriatric and intern wards is $10-31 \%$ [6]. It is estimated that the incidence of delirium among patients in intensive care units, on cardiac surgery and orthopedics wards is respectively $82 \%, 46 \%$ and $51 \%$. About $8-17 \%$ patients in emergency departments and $22 \%$ elderly patients of nursing homes were diagnosed with delirium [6].

Delirium is often confused with the behavioral and psychological symptoms of dementia (BPSD), depression and disorders secondary to "geriatric giants" or somatic illnesses. The fundamental characteristics of delirium are: a sudden beginning and impairment of cognitive functions such as fresh memory disturbances, confusion, or disorientation. Varying symptom intensity in time is typical.

The diagnostic criteria of delirium are listed by the authors of the ICD-10 and DSM classifications. Differences in the sensitivity and 
specificity between the ICD-10 and DMS-IV criteria was observed in trials to estimate the effectiveness and clinical applicability of those criteria. Applying dementia diagnostic criteria requires specialist knowledge and experience, therefore it should be used by psychiatrists and qualified doctors of other specializations [8-10].

Diagnosing delirium with those criteria is challenging for healthcare providers, commonly working with elderly patients as it is time-consuming and requires special interaction between the patient and healthcare provider. Therefore, it is often impossible in emergency states or during a hospital stay. The medical history and documentation provided by caregivers confirms the presence of "geriatric giants": depression, dementia, hearing or sight impairment, significant deterioration of physical performance, malnutrition, injuries, and urinary incontinence. Each of these conditions may lead to delirium.

Delirium may occur at every age, nevertheless, the incidence is greater in patients over 60 years old. The risk factors include male sex, concomitant diseases and polypharmacy (receiving many drugs at one time), depression, a history of delirium, stroke or other neurological condition and a history of alcohol abuse.

Delirium may be caused by surgery, acute coronary syndrome, head injuries, proximal femur fracture or immobilization [11]. Delirium predisposing factors include: medications with anticholinergic agents, opioids, benzodiazepines, nonbenzodiazepine hypnotics, dihydropyridines (e.g. nifedipine), antihistamine agents and presumably also with $\mathrm{H} 2$ agonists, tricyclic antidepressants, medications used in Parkinson disease treatment, steroids, diuretics and NSAIDs.

Other factors which may cause delirium are urinary tract infections, pneumonia, diverticulitis, respiratory distress (hypoxia, hypercapnia), urinary retention and catheterization, renal diseases, constipation and stool retention, undiagnosed or untreated pain, metabolic disorders such as hypoglycemia, dehydration or hyponatremia [11].

Predisposing factors also include stressful situations such as changing the environment, moving to the child's house or a nursing home, hospitalization, communication problems caused by language barriers or speech difficulties due to neurological impairment.

The diagnosis of delirium by non-qualified healthcare providers is facilitated by numerous psychometric and specialist tools and scales [12-15]. The most common are: CAM - Confusion Assessment Method commonly used in ICU and surgical wards [16]; MDAS - Memorial Delirium Assessment Scale [17]; DOSS Delirium Observation Screening Scale [18-20] and the recently verified tool $4 \mathrm{AT}$ - the rapid assessment test for delirium $[21,22]$. It easy to check that the forms of all enumerated scales can be found in appropriate Internet websites.

Trials have shown the usefulness of the 4AT test in emergency departments and during first contact with an elderly patient, as performing this test requires no special training and takes only 2 minutes. In just 4 steps healthcare providers evaluate: 1) alertness; 2) numbers of mistakes during the Abbreviated Mental Test; 3) attention with the Months Backwards Test and 4) a significant change or fluctuation in alertness, cognition or other mental function over the last 2 weeks and still evident in the last 24 hours. A result $\geq 4$ points is diagnostic of possible delirium with or without cognitive impairment. Proper diagnosis of delirium warrants optimization of care and stress reduction by formal and informal caregivers.

\section{Conclusion}

The awareness of delirium as a distinct disease, independent from dementia, among the population is low [10]. Therefore, the diagnosis of delirium is infrequent. Family caregivers equate delirium with dementia or psychical disorders. Up to $70 \%$ of patients who underwent delirium, remembers the episode and feels fear and embarrassment for that reason [10]. Taking into account the facts mentioned above in this article and the experience of qualified healthcare providers - doctors and nurses, along with family caregivers, it is possible to significantly improve the effectiveness of care of elderly patients in hospitals and nursing homes affected by delirium. They will therefore be able to quickly react to patients' behavioral changes.

Our article lists the minimum amount of knowledge necessary for every healthcare provider. Our purpose is to emphasize the education of healthcare providers in diagnostic methods, including the recently verified $4 \mathrm{AT}$ test and the principles of preventing delirium along with management of this condition.

\section{Acknowledgement}

We express our gratitude to Miss Christine Frank-Szarecka for the linguistic correction of the manuscript.

\section{Conflict of Interest}

No conflict of interest.

\section{References}

1. Witlox J, Eurelings LS, de Jonghe JF, Kalisvart KJ, Eikelenboom P, et al. (2010) Delirium in elderly patients and the risk of post discharge mortality, institutionalization, and dementia: a meta-analysis. JAMA 304(4): 443-451.

2. Francis J, Kapoor WN (1992) Prognosis after hospital discharge of older medical patients with delirium. J Am Geriatr Soc 40(6): 601-606.

3. Stevens LE, de Moore GM, Simpson JM (1998) Delirium in hospital: does it increase length of stay? Aust N Z J Psychiatry 32(6): 805-808.

4. Evensen S, Saltvedt I, Lydersen S, Wyller TB, Taraldsen K, et al. (2018) Environmental factors and risk of delirium in geriatric patients: an observational study. BMC Geriatr 18(1): 282.

5. Ahmed S, LeurentB, Sampson EL (2014) Risk factors for incident delirium among older people in acute hospital medical units: a systematic review and meta-analysis. Age Ageing 43(3): 326-333.

6. Siddiqi N, House AO, Holmes JD (2006) Occurrence and outcome of delirium in medical inpatients: a systematic literature review. Age Ageing 35(4): 350-364.

7. Oh ES, Fong TG, Hshieh TT, Inouye SK (2017) Delirium in Older Persons: Advances in Diagnosis and Treatment. JAMA 318(12): 1161-1174.

8. Lawlor PG, Bush SH (2014) Delirium diagnosis, screening and management. Curr Opin Support Palliat Care 8(3): 286-295.

9. WHO (1983) International Statistical Classification of Diseases and Health Related Problems, $10^{\text {th }}$ revision, Geneva. 
10. American Psychiatric Association (2013) Diagnostic and statistical manual of mental disorder $\left(\mathrm{V}^{\text {th }} \mathrm{edn}\right)$. American Psychiatric Association, Washington, USA

11. Grassi L, Caraceni A, Mitchell AJ, Nanni MG, Berardi MA, et al. (2015) Management of delirium in palliative care: a review. Curr Psychiatry Rep 17(3): 550

12. Hasemann W, Tolson D, Godwin J, Spirig R, Frei IA, et al. (2018) Nurses' recognition of hospitalized older patients with delirium and cognitive impairment using the delirium observation screening scale: A prospective comparison study. J Gerontol Nurs 44(12): 35-43.

13. van Velthuijsen EL, Zwakhalen SM, Warnier RM, Mulder WJ, Verhey FR, et al. (2016) Psychometric properties and feasibility of instruments for the detection of delirium in older hospitalized patients: a systematic review. Int J Geriatr Psychiatry 31(9): 974-989.

14. Hargrave A, Bastiaens J, Bourgeois JA, Neuhaus J, Josephson SA, et al. (2017) Validation of a Nurse-Based Delirium-Screening Tool for Hospitalized Patients. Psychosomatics 58(6): 594-603.

15. Wong CL, Holroyd-Leduc J, Simel DL, Straus SE (2010) Does this patient have elirium?: value of bedside instruments. JAMA 304(7): 779-786.

16. Flanagan NM, Spencer G (2016) Informal caregivers and detection of delirium in postacute care: a correlational study of the confusion assessment method (CAM), confusion assessment method-family assessment method (CAM-FAM) and DSM-IV criteria. Int J Older People Nurs 11(3): 176-183.

17. Barahona E, Pinhao R, Galindo V, Noguera A (2018) The Diagnostic Sensitivity of the Memorial Delirium Assessment Scale-Spanish Version. J Pain Symptom Manage 55(3): 968-972.

18. Gavinski K, Carnahan R, Weckmann M (2016) Validation of the delirium observation screening scale in a hospitalized older population. J Hosp Med 11(7): 494-497.

19. Teale E, Young J, Siddiqi N, Munyombwe T, Harrison J, et al. (2016) Study protocol-investigation of the Delirium Observation Screening Scale (DOSS) for the routine detection of delirium in the care home setting: a prospective cohort study. BMJ Open 6(6): e009615.

20. Jorgensen SM, Carnahan RM, Weckmann MT (2017) Validity of the Delirium Observation Screening Scale in Identifying Delirium in Home Hospice Patients. Am J Hosp Palliat Care 34(8): 744-747.

21. Shenkin SD, Fox C, Godfrey M, Siddiqi N, Goodacre S, et. al. (2018) Protocol for validation of the 4AT, a rapid screening tool for delirium: a multicentre prospective diagnostic test accuracy study. BMJ Open 8(2): e015572.

22. Bellelli G, Morandi A, Davis DH, Mazzola P, Turco R, et al. (2014) Validation of the 4AT, a new instrument for rapid delirium screening: a study in 234 hospitalised older people. Age Ageing 43(4): 496-502. 